\title{
The Optimal Consumption and the Quitting of Harmful Addictive Goods
}

\author{
Ruqu Wang* \\ Economics Department \\ Queen's University \\ Kingston, Ontario K7L 3N6 \\ Canada \\ e-mail:wangr@qed.econ.queensu.ca
}

March 22, 1999

*I am very grateful to two anonymous referees whose constructive suggestions improved the quality of the paper greatly. I would like to thank them, Marilyn Banting, Dan Bernhardt, Ryan Davies, Michelle Eggleston, Chris Ferrall, Devon Garvie, Alan Gunderson, Bart Lipman, Glenn Loury, Bob Rosenthal, Doug Tattrie, Andy Weiss, and JeanFrancois Wen for their very helpful comments. Financial support from the Social Sciences and Humanities Research Council of Canada is gratefully acknowledged. 


\begin{abstract}
In this paper we study a model of rational consumption and quitting in the context of harmful addictive goods. We assume that a person has imperfect information about his ability to resist and terminate the addiction. We first characterize the optimal consumption path of a non-addicted person, along which his stock of the addictive substance is either always increasing (and thus addiction occurs stochastically), always decreasing, or always unchanged. We then characterize the optimal consumption path of an addicted person, along which he may attempt to quit the addiction for a period of time, and then resume his consumption if the attempt is unsuccessful. Finally, we remark on the issues of regret, multiple attempts to quit, and quitting programs.
\end{abstract}

JEL Classification No.: C61, D11, D83, I12

Keywords: Rational Addiction, Quitting, Harmful Addiction, Imperfect Information 
There have been a series of attempts to analyze the behavior of smokers and other consumers of addictive goods. Harmful addictive goods such as alcohol, cocaine, and cigarettes, and harmful habits such as overeating and gambling receive most of the attention. Papers on rational addiction characterize an addicted consumer's consistent and rational use of the addictive substance. ${ }^{1}$ Papers on habit formation and habit persistence relate the short-run utility functions to the long-term utility functions. ${ }^{2}$ All of these papers assume that consumers know perfectly their short-run or long-run utilities of consuming the addictive good. The initial consumption is usually caused by some stressful event (marital breakup, peer pressure, job loss, etc.). A positive initial consumption would lead to addiction, since a larger past consumption increases the marginal utility of current consumption.

Four important aspects of addiction are difficult to explain with the above-mentioned models. First, since consumers can perfectly foresee their future, these models imply that addiction is a planned and desirable event. While consumers may plan their consumption of the addictive goods, in practice they never plan or enjoy getting addicted. Second, since everyone maximizes utility, no one ever has regrets in these models. In reality, many people $\underline{\text { do }}$ regret their consumption once they discover that they are addicted to the good. Third, since addiction is a planned event, no one wants to quit once they become addicted in these models. This is contradicted by the fact that most people try very hard to terminate the addiction later in life. Fourth, according to these models, if for whatever reasons people decide to quit (either "cold turkey" or by other less abrupt methods), they succeed. The fact that many people try to quit, but fail, is then difficult to explain. ${ }^{3}$

These stylized facts highlight the seemingly intertemporal time inconsistency in the behaviors of these consumers. In a forward-looking, perfect information, rational expectation model, consumers know exactly what to expect, and a change of course in the middle of the consumption path is inconsistent with the assumption of utility maximization.

We argue that, to some extent, the difficulties in explaining these key behaviors of addicts are a result of the lack of uncertainty in the models. To most people, addictions do not occur with

\footnotetext{
${ }^{1}$ See Stigler and Becker (1977), Becker and Murphy (1988), Becker, Grossman and Murphy (1991), Michaels (1988), and Barthold and Hochman (1988). Chaloupka's (1991) estimates imply that smokers are rational. Viscusi's (1990) study shows surprisingly that smokers in general overestimate the risk of health consequences of smoking. Consumers in this paper are assumed to be rational and forward looking.

${ }^{2}$ See Pollak (1970, 1976), Spinnewyn 1981, Iannaccone (1984, 1986) and Bordley (1986).

${ }^{3}$ Becker and Murphy (1988) try to explain the phenomenon of failed quitting attempts by going outside their model. Dockner and Gustav (1993) explain this kind of cyclical consumption pattern by using two interacting states, one of which is addictive and the other is satiating.
} 
certainty. As Brown (1986, p.636) put it, "[a]ctions like smoking a cigarette, having a drink, eating a candy bar, and working overtime to 'catch up' all lead to immediate and certain gratification, whereas their bad consequences are remote in time, only probabilistic, and still avoidable now." This uncertain aspect of addiction has been recognized by Orphanides and Zervos (1995), who studied the behavior of drug experimentation in a discrete-time model. In their model, consumers did not know whether they were potentially addicted to a good, but could find out (with some positive probability) by consuming a positive amount of it. As a result, learning and regret are explained within a rational expectation framework.

This paper incorporates uncertainty in a continuous-time rational expectation model. This uncertainty aspect is similar to that in the discrete-time model in Orphanides and Zervos (1995). Therefore, both explain regrets and the randomness of addiction. In their model, a person is uncertain whether or not the good is harmful and addictive to him, and can find out (with some probability) by experimenting with a small amount of that good. If the good is harmful, a BeckerMurphy type model follows and addiction is defined accordingly. In our model, the good is harmful to a person if he accumulates a stock of the addictive substance exceeding a previously unknown threshold level. Therefore, addiction occurs stochastically, and regrets can happen in both models. There are many important distinctions between the two models, however. Because of the differences in uncertainty, a person never quits in the Orphanides-Zervos model but he sometimes quits in our model. Consequently, a person's quitting behavior is part of the focus of this study but not in their paper. We assume that an addicted person can terminate his addiction by reducing his stock of the addictive substance below another previously unknown threshold level. Therefore, a person may fail in his attempt to quit because of the uncertainty. In all Becker-Murphy type models, however, once a person decides to quit, he succeeds. In addition, the Orphanides-Zervos approach cannot be generalized directly to a continuous-time setting, since a person would continuously experiment with a very small amount of the good and determine his tendency towards addiction almost immediately; this does not happen in our model. Finally, everyone in our model is an addict if he consumes sufficient amount of the addictive good, while in the Orphanides-Zervos model some are addicts (but not others) independent of how much they consume.

The major assumption we make in the analysis is that a person obtains uniformly less utility when he is addicted than when he is not. Therefore, we treat addiction as a state of harm, instead of something measured by how much a person consumes around the steady state (as in Becker and Murphy (1988) and Orphanides and Zervos (1995)). Once the person enters this state, his utility drops suddenly (given the same consumption and the same stock of the addictive good), until he is no longer addicted. The uncertainty regarding the two above-mentioned threshold levels is a recognition of the fact that different people have different abilities to resist the influence of 
addictive substances and different experiences in quitting. Some people are able to consume large quantities of an addictive good without becoming addicted while others are not. Likewise, some addicted people can quit easily, but others cannot.

This paper employs techniques for analyzing optimization problems under state-dependent uncertainty. Similar techniques have been used in investigating the optimal depletion of extractable resource of unknown quantity (see Dasgupta and Heal (1974)), the optimal investment decision in the presence of uncertain future changes (see Nickell (1978, pp. 93-105)), and the optimal consumption and life insurance when a consumer has an uncertain lifetime (see Yaari (1965)). These techniques can also be applied to other problems such as machine maintenance and government spending (deficits and credit ratings), where discrete jumps in value occur stochastically and are dependent on the current state of the system.

The analysis will proceed as follows. In Section I, the model is introduced, the overall optimization problem is partly analyzed, and the optimal consumption path of a non-addicted person is developed. In Section II, the techniques are applied to the optimal quitting of an addicted person, with a subsection devoted to a comparison between the consumption in the consumption model and in the quitting model. In Section III, remarks are given on the relationship with other preference switching models in the literature, the issue of regret, the phenomenon of multiple attempts to quit, and the consequences of easier quitting. In Section IV, the conclusions are summarized.

\section{The Consumption of Harmful Addictive Goods}

Consider a rational person who lives forever. His utility from consuming an addictive good depends on both his accumulation of the addictive substance and whether he is addicted to the good at the time of consumption. Let $c(t)$ be his consumption of the addictive good at time $t$ and $S(t)$ be the stock of the addictive substance he accumulates by $t$. It is assumed that the accumulated stock depreciates at rate $\delta$. Thus the change in his stock is given by

$$
\dot{S}(t)=c(t)-\delta S(t)
$$

where $c(t) \geq 0, \forall t \geq 0$.

The person's utility function is separable and additive. Suppose that he consumes $c$ when his

stock is $S$. If he is not addicted at the time of consumption, his utility is $U(c, S)$. If he is addicted, however, his utility is $\mathcal{U}(c, S)$, where

$$
U(c, S)>\mathcal{U}(c, S), \quad \forall c, \forall S
$$

Hence, addiction is harmful to the person. Throughout this paper, we restrict $c$ to be a piecewise continuous function, and assume that $U(\cdot, \cdot)$ and $\mathcal{U}(\cdot, \cdot)$ are both strictly concave and continuously 
three times differentiable.

In general, a person's utility also depends on his consumption of other goods. If a person has a fixed income and takes the prices of the addictive good and other goods as given, the above described utility functions can be regarded as reduced-form utility functions. This is similar to equation (7) in Becker and Murphy (1988), where the person optimizes other consumption given the consumption of the addictive good. The above utility functions can also be justified in a manner similar to Orphanides and Zervos (1995), where they assume that the budget constraint is binding every period; if one consumes $c$ units of the addictive good, then the rest of his income is spent on normal goods. Therefore, a person's utility can be expressed solely by his consumption on the addictive good. As an important implication, $U_{c}>0$ and $\mathcal{U}_{c}>0$ are not always guaranteed in this paper. (However, we do impose some conditions on $U$ and $\mathcal{U}$ later.)

Notice that the person's utility depends not only on how much he consumes, but also on his stock of the addictive substance at the time of consumption. Therefore, it characterizes the effect of past consumption on the utility of current consumption.

There are two threshold levels of the stock of this addictive substance, $\bar{S}$ and $\underline{S}$, that are important to our analysis. $\bar{S}$ is the threshold level above which a non-addicted person becomes an addict, and $\underline{S}$ is the threshold level below which an addict can terminate his addiction and become a non-addicted person once more. It is logical to assume that $\bar{S}>\underline{S}$. A situation where $\bar{S} \leq \underline{S}$ would indicate that sometimes a person is both an addict and a non-addict, thereby implying an inconsistency over the state of addiction in our formulation.

A person does not have perfect information about $\bar{S}$ and $\underline{S}$. The exact value of $\bar{S}$ is unknown until the moment the person (being non-addicted) increases his stock $S$ to $\bar{S}$ and immediately becomes addicted. Similarly, the exact value of $\underline{S}$ is unknown until the addicted person actually decreases his stock $S$ to $\underline{S}$ and becomes a non-addicted person again. A priori, $\bar{S}$ follows the distribution characterized by c.d.f. $G(\cdot)$ and p.d.f. $g(\cdot)$, with support $[0, \infty)$. The unconditional $a$ priori distribution regarding $\underline{S}$ is given by $F(\cdot)$, with support $[0, \infty)$. Given that a person becomes addicted at $\bar{S}$, however, the belief about $\underline{S}$ must be adjusted, since a person's $\underline{S}$ is always lower than his $\bar{S}$. Given that $\bar{S}$ is known, the distribution of $\underline{S}$ is given by c.d.f. $F(\cdot) / F(\bar{S})$ and p.d.f. $f(\cdot) / F(\bar{S})$, with support $[0, \bar{S}]$. The process of quitting is characterized by $\dot{S}<0$. Given that the person is still addicted, his probability assessment on $\underline{S}$ with current stock $S \leq \bar{S}$ is conditional on $\underline{S}<S$ and given by c.d.f. $F(\cdot) / F(S)$, with support $[0, S]$. Throughout this paper, we assume that $f$ and $g$ are continuous and strictly positive on their respective supports.

Note that in this paper, everyone is an addict when his stock of the addictive substance exceeds his limit. This limit, however, is different from person to person. Therefore, one non-addicted person could consume more than some other addicted person. A specific person could also consume 
more when he is not addicted than when he is, even though it might not be optimal. Under the assumptions in this paper, however, he always consumes more (in the long run) when he is addicted than when he is not, along the optimal path. Subsection (D) in Section II analyzes this situation.

Suppose that a stressful event occurs at the beginning of a person's life. The consumption of the addictive good gives the person temporary relief, but also drives up his initial stock of the addictive substance to $S_{0}$. This stock, $S_{0}$, is zero if there is no stressful event or if he decides not to consume the addictive good even if there is a stressful event. In this section, we consider the case where the person is not addicted given this initial stock (i.e., $S_{0}<\bar{S}$ ). (If he is initially addicted, he has to decide whether or not to quit the addiction. This case is analyzed in Section II.)

A person's history is important in maximizing his expected utility. At each moment, a person knows whether or not he is addicted and how much he consumes. He does not forget what he knew in the past and thus can calculate his current stock of the addicted substance according to (1). Let $I$ be the indicator function that equals zero if he is not addicted and 1 if he is. $I$ is right-continuous, and it is similar to the hazard or cumulative-risk function in the stochastic process literature (see, for example, Rogers and Williams (1994, pp. 167-169; 1987, pp. 352-354)). The cumulative risk function has many economic applications, including the pricing of defaultable bonds (see, for example, Duffie and Singleton (1998)).

Let $I^{-}(t)=\lim _{x \rightarrow t^{-}} I(x), \forall t>0$. This limit always exists. ${ }^{4}$ The transition of the addiction indicator $I$ can be written as, $\forall t>0$,

$$
I(t)=\left\{\begin{array}{cc}
0, & \text { if } S(t)=\underline{S} \text { and } I^{-}(t)=1 \\
1, & \text { if } S(t)=\bar{S} \text { and } I^{-}(t)=0 \\
I^{-}(t), & \text { otherwise. }
\end{array}\right.
$$

Given this indicator function, a person's utility at $t$ can be written as

$$
u(c(t), S(t), I(t))=(1-I(t)) U(c(t), S(t))+I(t) \mathcal{U}(c(t), S(t)) .
$$

Two moments are important in a person's information updating. Consider a specific path of consumption. Let $t_{a}=\inf \{\tau: I(\tau)=1\}$ and $t_{q}=\inf \left\{\tau>t_{a}: I(\tau)=0\right\} . t_{a}$ is the first time the person became addicted and $t_{q}$ is the first time he terminated the addiction. Note that these values may not exist (treat them as $\infty$ if so), and if they do, they are uniquely determined. Also, a person may become addicted and quit many times in his life. Nonetheless, after $t_{q}$, all information regarding his addiction and quitting tendencies becomes perfect information, and it may not be optimal to become addicted again.

\footnotetext{
${ }^{4}$ This is because $S$ is always continuous and $\dot{S}$ is bounded from below by $-\delta S$. Therefore, within a given amount of time, $S$ could cross $\bar{S}$ and then $\underline{S}$ for at most finitely many times. This implies that $I$ is piecewise continuous and $I^{-}$always exists.
} 
Define $\vec{S}(t)=\sup \{S(\tau): \tau \leq t\}$ as the maximal stock of the addictive substance in a person's history up to time $t .^{5}$ Define also $\breve{S}(t)=\inf \left\{S(\tau): t_{a} \leq \tau \leq t\right\}$ as the lowest stock up to time $t$ since his first addiction. Using these two stocks, the person can update his posterior belief on $\bar{S}$ and $\underline{S}$ accordingly.

If $t<t_{a}$, then the person has never been addicted and thus $\bar{S}>\vec{S}(t)$. Therefore, the conditional probability for $\bar{S}<S$ becomes $\frac{G(S)-G(\vec{S}(t))}{1-G(\vec{S}(t))}$, where $S \in(\vec{S}(t), \infty)$. Conditional upon $\bar{S}$, the c.d.f. for $\underline{S}$ remains unchanged. If $t \in\left[t_{a}, t_{q}\right)$, then the person has been addicted but has not yet terminated his initial addiction. Therefore, he knows $\bar{S}$ perfectly and $\underline{S}$ imperfectly; that is, $\operatorname{Pr}\left\{\bar{S}=S\left(t_{a}\right)\right\}=1$ and $\underline{S}<\breve{S}(t)$. In this case, the conditional probability for $\underline{S}<S$ is given by $\frac{F(S)}{F(\breve{S}(t))}$, where $S \leq \breve{S}(t)$.

At $t=t_{q}$, the person quits his addiction successfully after having been addicted in the past. Therefore, $\bar{S}$ and $\underline{S}$ are now known perfectly and $\operatorname{Pr}\left\{\bar{S}=S\left(t_{a}\right)\right\}=\operatorname{Pr}\left\{\underline{S}=S\left(t_{q}\right)\right\}=1$. What a person does after quitting could be quite different from his earlier behavior, since now he has perfect information about both $\bar{S}$ and $\underline{S}$. Denote $\mathcal{W}(S)$ as the present value of the person's maximized utility if he quits the addiction exactly at $S$ (which is equal to his $\underline{S}$ ). Of course, this $\mathcal{W}(S)$ depends on the value of $\bar{S}$, which is omitted here to save notation. In this paper, we shall not investigate $\mathcal{W}(S)$, except to assume that quitting successfully is better than still being addicted in the second part of our analysis.

Given $\bar{S}$ and $\underline{S}$, we can calculate $t_{a}$ and $t_{q}$ along any consumption path $c$ and the corresponding state path $S$. Given $t_{a}$ and $t_{q}$, we can express the consumer's utility as

$$
\begin{aligned}
& \int_{0}^{\infty} u(c(t), S(t), I(t)) e^{-\rho t} d t \\
= & \int_{0}^{t_{a}} U(c(t), S(t)) e^{-\rho t} d t+\int_{t_{a}}^{t_{q}} \mathcal{U}(c(t), S(t)) e^{-\rho t} d t+\int_{t_{q}}^{\infty} u(c(t), S(t), I(t)) e^{-\rho t} d t
\end{aligned}
$$

Using the principle of dynamic optimization and the above defined $\mathcal{W}(\cdot)$, this person's optimization problem can be written as follows:

$$
\begin{gathered}
\max E_{0}\left[\int_{0}^{t_{a}} U(c(t), S(t)) e^{-\rho t} d t+\int_{t_{a}}^{t_{q}} \mathcal{U}(c(t), S(t)) e^{-\rho t} d t+\mathcal{W}\left(S\left(t_{q}\right)\right) e^{-\rho t_{q}}\right] \\
\text { s.t. } \dot{S}=c-\delta S, \quad S(0)=S_{0}, \quad I(0)=0,
\end{gathered}
$$

where the expectation is taken with respect to $\underline{S}$ and $\bar{S}$ at time $t=0$, and $t_{a}$ and $t_{q}$ are dependent on $\underline{S}, \bar{S}$, and the consumption path.

The above formulation can be used to investigate some aspects of the addiction problem, but not others. For example, we assumed that the person lives forever. This simplifying assumption

\footnotetext{
${ }^{5}$ Note that $S$ is always continuous and the supremum is equal to the maximum.
} 
is obviously not a very realistic one. It would be more realistic to allow death in the model. If death occurs exogeneously and stochastically, and follows a Poisson process, we can easily take it into account by adding the death rate to the economically motivated discount rate. However, since addiction is harmful, it may alter a person's life span endogeneously. A person who consumes a large amount of this addictive good may have a significantly shorter life. Unfortunately, the relatively simple analysis in this paper is not general enough to address this issue.

The assumption that addiction is harmful, $\mathcal{U}(c, S)<U(c, S), \forall c, \forall S$, implies that a person does not like being addicted. However, the consumption of the addictive good produces temporary satisfaction. Moreover, consuming a small amount of the good does not always lead to addiction, since the person's threshold level $\bar{S}$ may be quite high. Therefore, a non-addicted person may rationally consume the addictive good and risk the possibility of becoming addicted. On the other hand, because addiction lowers utility, there is still a limit to the addictive stock a non-addicted person would like to optimally accumulate, even if the person is risk neutral.

Along any feasible consumption path, a person may sometimes consume more and sometimes consume less. Sometimes a person faces the risk of addiction and sometimes not. It is not easy to compare these paths and select the optimal one. Fortunately, we find that given the information structure in our model, the optimal path can be qualitatively characterized by considering the person's behavior before and after his first addiction separately. We have the following lemma:

Lemma 1 The optimal consumption path must fall into one (and only one) of the following cases:

1. $\dot{S}(t) \leq 0$, for $t \geq 0$;

2. There exists a $\tilde{T}<t_{a}$, such that $\dot{S}(t)>0$ for $\tilde{T}>t \geq 0$, and $\dot{S}(t) \leq 0$ for $t \geq \tilde{T}$.

3. $\dot{S}(t)>0$, for $0 \leq t<t_{a}$, and there exists a $T \in\left[t_{a}, \infty\right) \cup\{\infty\}$, such that

a) if $t_{q} \leq T$, then $\dot{S}(t)<0$ for $t_{a}<t<t_{q}$;

b) if $t_{q}>T$, then $\dot{S}(t)<0$ for $t<T$ and $\dot{S}(t)>0$ for $t>T$.

Proof First, we consider the person's consumption path after he becomes addicted for the first time, but before he quits successfully, that is, when $t_{a}<t<t_{q}$. Pick any such $t$. When $S(t)=\breve{S}(t)$ and $c(t)<\delta S(t), \dot{S}(t)$ is negative, and the information on $\underline{S}$ is updated. Otherwise, the information is unchanged. Suppose that at some $t \in\left(t_{a}, t_{q}\right)$, the person is still addicted, and $c(t)>\delta S(t)$ is optimal. Then at the first $\tilde{t}>t$ with the property $S(\tilde{t})=S(t), c(\tilde{t})>\delta S(\tilde{t})$ is still optimal, since the information on $\underline{S}$ (and anything else) is the same as before. Apply this principle to all $\tau \in(t, \tilde{t})$ and we can conclude that there should not exist a $\tau$ with $\dot{S}(\tau)<0$. This implies that if $S$ starts to increase, it should not decrease afterwards. It leads to three possibilities for the consumption 
path between first addiction and its first successful termination: $S$ is always increasing, $S$ is always decreasing, or $S$ is first decreasing and then increasing. Define $T=\inf \left\{t: c(t)>\delta S(t), t \geq t_{a}\right\}$, and set its value to $\infty$ if such $t$ does not exist. The person's optimal behavior after $t_{a}$ is thus proved.

Now we consider the person's optimal consumption path before his first addiction. His information at $t$ regarding $\bar{S}$ is given by $\bar{S} \geq \vec{S}(t)$. When $S(t)=\vec{S}(t)$ and $c(t)>\delta S(t), \dot{S}(t)$ is positive, and the information on $\bar{S}$ is updated. Otherwise, the information is unchanged. Suppose that at some $t<t_{a}, c(t)<\delta S(t)$ is optimal. Then at the first $\tilde{t}>t$ with the property $S(\tilde{t})=S(t)$, $c(\tilde{t})<\delta S(\tilde{t})$ is still optimal, since the information on $\bar{S}$ (and anything else) is the same as before. Apply this principle to all $\tau \in(t, \tilde{t})$ and we can conclude that there should not exist a $\tau \in(t, \tilde{t})$ with $\dot{S}(\tau)>0$. This implies that if $S$ starts to decrease, it should not increase afterwards. It leads to three possibilities for the optimal path before his first addiction: $S$ is always increasing, $S$ is always decreasing, or $S$ is increasing first and then decreasing later (denote the dividing moment as $\tilde{T}$ in this case).

The lemma is proved by combining the possibilities in the two segments of the consumption path.

Note that Lemma 1, we characterize the optimal consumption path up to $t_{q}$ (if it exists). We simply assume that quitting successfully is better than still being addicted. Case 2 in this lemma never occurs in our model because addiction is harmful: a person would not risk becoming addicted by consuming a lot, find out that he is not yet addicted, and then consume less. (This will become clear later in the analysis.) Furthermore, a person's stock of the addictive substance will not be switching back and forth between any two values along the optimal consumption path. Such cyclical behavior occurs only in models with more than one state variable (c.f. Dockner and Feichinger (1993)); in our model, the stock of the addictive substance is the only state variable.

We shall analyze the person's optimal consumption problem in two sections. In this section, we investigate the person's optimal consumption before his first addiction, leaving the optimal consumption problem after his first addiction to the next section.

The following two assumptions are made throughout this paper.

$\underline{\text { Assumption } 1} \quad(\delta+\rho) U_{c}(0,0)+U_{S}(0,0) \geq 0$.

Assumption $2 \quad \delta U_{c c}(c, S)+U_{c S}(c, S)<0, \forall c \geq 0, \forall S \geq 0$.

Assumption 1 ensures that the person would prefer to consume some amount of the addictive good given that there is no risk of addiction. The person may not consume anything at all if there 
is some risk that his utility will be reduced to $\mathcal{U}$. Assumption 2 together with the concavity of $U(\cdot, \cdot)$ implies that $(\delta+\rho) U_{c}(\delta S, S)+U_{S}(\delta S, S)$ is a decreasing function of $S$. This is because

$$
\begin{aligned}
& \frac{d}{d S}\left[(\delta+\rho) U_{c}(\delta S, S)+U_{S}(\delta S, S)\right] \\
= & (\delta+\rho)\left[\delta U_{c c}(\delta S, S)+U_{c S}(\delta S, S)\right]+\delta U_{c S}(\delta S, S)+U_{S S}(\delta S, S) \\
= & \rho\left[\delta U_{c c}(\delta S, S)+U_{c S}(\delta S, S)\right]+\left[\delta^{2} U_{c c}(\delta S, S)+2 \delta U_{c S}(\delta S, S)+U_{S S}(\delta S, S)\right]<0 .
\end{aligned}
$$

The last inequality holds because of the following. From Assumption 2, the terms in the first pair of square brackets are negative. From the assumption of concavity of $U(\cdot, \cdot)$, the terms in the second pair of brackets are negative.

As we shall see later, these Assumptions 1 and 2 guarantee that the steady state of the nonaddiction part of the system here has a unique saddle point $\left(c^{*}, S^{*}\right)$, with $c^{*} \geq 0$ and $S^{*} \geq 0$. Therefore, our system here is quite different from the unstable system in Becker and Murphy $(1988){ }^{6}$

Let us first look at the case when $c \leq \delta S$ is imposed. In this case, $S$ is decreasing along any feasible path. Obviously, there is no risk of addiction along this path, as the person is not addicted at $t=0$ which has the highest $S$ along the path. Therefore, the only relevant utility function is $U$. The person chooses $c$ to maximize the present value of his expected utility:

$$
\begin{gathered}
V\left(S_{0}\right)=\max \int_{0}^{\infty} U(c(t), S(t)) e^{-\rho t} d t . \\
\text { s.t. } \quad \dot{S}=c-\delta S, \quad 0 \leq c \leq \delta S, \quad S(0)=S_{0} .
\end{gathered}
$$

We first ignore the constraint on $c$. Let $H=U(c, S)+\lambda(c-\delta S)$ be the current value Hamiltonian for this maximization. We have the optimality condition $H_{c}=0$, and the co-state equation $H_{S}=$ $\rho \lambda-\dot{\lambda}$. Solving $H_{c}=0$ and $H_{S}=\rho \lambda-\dot{\lambda}$, the co-state equation can be rewritten as

$$
\dot{c}=\frac{1}{U_{c c}}\left[(\delta+\rho) U_{c}+U_{S}-U_{c S}(c-\delta S)\right] .
$$

Let the steady-state stock of the addictive substance be $S^{*}$. Then $\forall t, S(t)=S^{*}$ and $c(t)=\delta S^{*}$ must satisfy $\dot{c}=0$ and $\dot{S}=0$. This implies

$$
(\delta+\rho) U_{c}\left(\delta S^{*}, S^{*}\right)+U_{S}\left(\delta S^{*}, S^{*}\right)=0 .
$$

\footnotetext{
${ }^{6}$ Becker and Murphy (1988) have multiple (stable and unstable) steady states.
} 
From Assumption 1 and inequality (2), the solution to the above equation is unique and nonnegative. Using Taylor expansion on the right-hand side of (3) around the steady state $\left(\delta S^{*}, S^{*}\right)$, and noting that the state equation is linear in $c$ and $S$, this dynamic system can be approximated around its steady state as

$$
\left(\begin{array}{c}
c-\delta S^{*} \\
S-S^{*}
\end{array}\right)^{\prime}=\left(\begin{array}{cc}
\frac{1}{U_{c c}}(\delta+\rho) U_{c c} & \frac{1}{U_{c c}}\left[(2 \delta+\rho) U_{c S}+U_{S S}\right] \\
1 & -\delta
\end{array}\right)\left(\begin{array}{c}
c-\delta S^{*} \\
S-S^{*}
\end{array}\right)
$$

From (2), the determinant of the coefficient matrix of this system is negative, implying that the steady state is a saddle point in the phase diagram in $(c, S)$. Therefore, a continuous saddle path for the original system (before approximation) exists, and given our assumptions, it is the solution to the infinite horizon autonomous problem (without the constraint on $c$ ). (See Seierstad and Sydsæter (Thm. 19, pp. 256-260, 1987) and Leonard and Van Long (Section 9.5 pp. 294-297, 1992) for details.) Let this optimal path be $c(t)=c^{*}(S(t)), \forall t$, where $c^{*}(\cdot)$ is continuous. Since $c(\cdot)$ and $S(\cdot)$ are the solutions to the simultaneous differential equations, they must be differentiable as well. Since $S^{\prime} \neq 0$ except at the steady state (i.e., when $t$ goes to infinity), we can also conclude that $c^{*}$ is differentiable.

Because $S$ converges to $S^{*}$ along this path as $t$ goes to infinity, it must be that when $S>S^{*}$, $\dot{S}<0$, and when $S<S^{*}, \dot{S}>0$. Stated equivalently, when $S>S^{*}, c<\delta S$, and when $S<S^{*}$, $c>\delta S$. Note that the path never crosses $c=\delta S$ except at $S^{*}$ because the solution to (4) is unique.

We argue that the part of $S(t)$ that satisfies $S(t) \geq S^{*}, \forall t$, must be the optimal path for $S_{0} \leq S^{*}$ when $c \leq \delta S$ is imposed. This is because it satisfies the constraint on $c$ and it is better than any other path using utility $U(\cdot, \cdot)$. Obviously, it must be better than any path that sometimes obtains the lower utility $\mathcal{U}(\cdot, \cdot)$.

If $S_{0}<S^{*}$, then $c(t)=c^{*}(S(t)), \forall t$, is not feasible anymore when $c \leq \delta S$ is imposed. In this case, we argue that the optimal path is given by $c \equiv \delta S_{0}$. From $c \equiv \delta S_{0}$, we have $S(t) \equiv S_{0}$. Note that the constant function $\lambda=U_{S}\left(\delta S_{0}, S_{0}\right) /(\rho+\delta)$ is a solution to the co-state equation $H_{S}=U_{s}-\delta \lambda=\rho \lambda-\lambda^{\prime}$. Given this $\lambda, H_{c}=U_{c}+\lambda=U_{c}\left(\delta S_{0}, S_{0}\right)+U_{S}\left(\delta S_{0}, S_{0}\right) /(\rho+\delta)>0$ for $S_{0}<S^{*}$ from (2) and (4). Therefore, the constraint $c(t) \leq \delta S(t)=\delta S_{0}$ is binding for every $t$. With $c(t) \leq \delta S(t)$ imposed, $S$ is bounded along any feasible path. This implies that the optimal path must be the corner solution $c=\delta S_{0}$. (c.f. Leonard and Van Long, Section 9.5 pp. 294-297, 1992)

We summarize the above results in the following lemma:

Lemma 2 Suppose that $c \leq \delta S$ is imposed in the optimization problem. Then the optimal consumption path is given by the saddle path $c(t)=c^{*}(S(t))$ (where $c^{*}(\cdot)$ is differentiable), $\forall t$, if $S_{0} \geq S^{*}$, and by $c(t)=\delta S_{0}, \forall t$, if $S_{0}<S^{*}$. 
Next we consider the general case where the person can choose to consume any amount he desires. If a person reaches his $\bar{S}$ at $t_{a}$, his utility can be divided into two parts:

$$
\int_{0}^{\infty} u(c(t), S(t), I(t)) e^{-\rho t} d t=\int_{0}^{t_{a}} U(c(t), S(t)) e^{-\rho t} d t+\int_{t_{a}}^{\infty} u(c(t), S(t), I(t)) e^{-\rho t} d t
$$

where $S\left(t_{0}\right)=S_{0}$ and $I\left(t_{a}\right)=1$. Let $\tilde{V}\left(S_{0}\right)$ be the maximized value of the above expression. It is the person's optimal expected utility when he is not addicted at the initial $S\left(t_{0}\right)=S_{0}$. Similarly, let $W(S)$ be the optimal current values of the person's discounted utility from the moment he becomes addicted (at stock $S$ ).

Because addiction is harmful, it is easy to see that

$$
\tilde{V}(S)>W(S), \quad \forall S \geq 0
$$

that is, facing the risk of addiction is definitely worse than actually being addicted. If the above inequality is reversed, it is called a healthy addiction; this situation is not considered in this paper.

Using the newly defined functions, we can restate the person's optimization problem using the principle of dynamic optimization:

$$
\tilde{V}\left(S_{0}\right)=\max \left\{\int_{0}^{t_{a}} U(c(t), S(t)) e^{-\rho t} d t+W\left(S\left(t_{a}\right)\right) e^{-\rho t_{a}}\right\} .
$$


To characterize the optimal consumption path, we use the method of dynamic programming. Suppose that the person consumes at a rate equal to $c$ from $t$ to $t+d t$ and the change in $S$ is given by $d S=(c-\delta S) d t$. First consider $d S>0$. Given that the person is not yet addicted at $S$, which happens with probability $1-G(S)$, the probability that he reaches his $\bar{S}$ by $S+d S$ is given by $\frac{G(S+d S)-G(S)}{1-G(S)}=\frac{g(S) d S}{1-G(S)}$. If $d S \leq 0$, then there is no risk of addiction. According to Lemma 1 , the optimal path will preserve this property $d S \leq 0$ from then on. The Hamilton-Jacobi-Bellman optimality equation can be written as:

$\tilde{V}(S)=\max _{c} \begin{cases}U(c, S) d t+e^{-\rho d t}\left[\tilde{V}(S+d S)\left(1-\frac{g(S) d S}{1-G(S)}\right)+W(S+d S) \frac{g(S) d S}{1-G(S)}\right], & \text { if } c \geq \delta S ; \\ U(c, S) d t+e^{-\rho d t} V(S+d S), & \text { if } c \leq \delta S .\end{cases}$

In (6), if $c-\delta S \geq 0$, then $d S \geq 0$. Note that $\frac{g(S) d S}{1-G(S)}$ is the probability that a person would first become addicted between $t$ and $t+d t$ when his stock increases from $S$ to $S+d S$, given that he is not yet addicted at $S$. If that event occurs, the person gets $W(S+d S)$. Otherwise, he gets $\tilde{V}(S+d S)$ again. Therefore, a person risks the danger of being addicted after consumption. If $c-\delta S \leq 0$, then $d S \leq 0$. According to Lemma 1, this property will be preserved along the optimal path. Because there is no risk of addiction in this case, the present value of the person's utility is given by $V(S)$. Note that when $c=\delta S$ is the optimal consumption, the two expressions in (6) are in fact the same.

Since the right-hand side of (6) represents the expected utility of consuming $c$ for a very short period of time $(d t)$, the optimal $c$ is given by the solution to the maximization in (6). There are two cases, depending on the optimal consumption $c$. If the optimal $c \leq \delta S$, then $\tilde{V}(S)=V(S)$. Otherwise, $\tilde{V}(S)>V(S)$.

Suppose that $c>\delta S$. So $\tilde{V}(S)>V(S)$. From Stokey and Lucas (Theorem 9.8, p. 265, and Theorem 9.10, p. 266, 1989), $\tilde{\mathcal{V}}$ is concave and continuously differentiable. Rearranging (6), ignoring all higher-order terms, and making use of $\frac{d S}{d t}=c-\delta S, e^{-\rho d t}=1-\rho d t, \tilde{V}(S+d S)=\tilde{V}(S)+\tilde{V}^{\prime}(S) d S$ and $W(S+d S)=W(S)+W^{\prime}(S) d S$, we have

$$
\rho \tilde{V}(S)=\max _{c \geq \delta S}\left\{U(c, S)+\left[\tilde{V}^{\prime}(S)-(\tilde{V}(S)-W(S)) \frac{g(S)}{1-G(S)}\right](c-\delta S)\right\} .
$$

The right-hand side of (7) captures the instantaneous effect of consumption. $U(c, S)$ is the instantaneous utility of consuming $c . \tilde{V}^{\prime}(S)(c-\delta S)$ is the change in future value due to an increase in $S$, while $(\tilde{V}(S)-W(S)) \frac{g(S)}{1-G(S)}(c-\delta S)$ measures the potential loss in utility because of the slight chance of becoming addicted. The total effect must be maximized along the optimal path, which 
yields the following first-order condition for any interior solution:

$$
U_{c}(c, S)+\tilde{V}^{\prime}(S)-(\tilde{V}(S)-W(S)) \frac{g(S)}{1-G(S)}=0 .
$$

It is easy to see that the sufficient second-order condition is always satisfied because $U_{c c}<0$. Combining (7) and (8), we have the necessary condition which the interior part of the optimal consumption must satisfy:

$$
\rho \tilde{V}(S)=U(c, S)-U_{c}(c, S)(c-\delta S)
$$

Even though this condition contains the unsolved value function, it has useful implications which are important to the characterization of the optimal consumption path. Consider an $S \geq S^{*}$. We claim that $c=\delta S$ must be the optimal consumption. This is because at $c=\delta S, \tilde{V}(S)=U(\delta S, S) / \rho$ (according to (9)). Therefore, the left-hand side of (8) becomes

$$
U_{c}(\delta S, S)+\frac{1}{\rho}\left[\delta U_{c}(\delta S, S)+U_{S}(\delta S, S)\right]-(\tilde{V}(S)-W(S)) \frac{g(S)}{1-G(S)} .
$$

From Assumptions 1 and 2, the first two terms together are negative for $S \geq S^{*}$. The last term is negative due to the assumption of harmful addiction. Therefore, the left-hand side of (8) must also be negative for $S \geq S^{*}$. This implies that the corner solution $c=\delta S$ is indeed optimal.

This result is quite intuitive. When $S \geq S^{*}$, the optimal consumption calls for $c \leq \delta S$ even if there is no risk of addiction. Now with the risk of addiction, the person must consume even less. Bounded by the restriction of $c \geq \delta S$, the corner solution $c=\delta S$ is obviously optimal.

Note that $c=\delta S$ may still be optimal under the constraint $c \geq \delta S$ even when $S<S^{*}$, as long as the left-hand side of $(8)$ is still negative. Let $\tilde{S}^{*}$ be the value that divides the two different types of consumption patterns with the following properties. For $S<\tilde{S}^{*},(8)$ is satisfied and an interior solution exists. Denote this solution as $c(t)=\tilde{c}(S(t)), \forall t$. From (8), it is easy to see that $\tilde{c}(\cdot)$ is continuous. For $S>\tilde{S}^{*},(8)$ does not hold and the corner solution $c=\delta S$ is the optimal consumption. We call this $\tilde{S}^{*}$ the person's target stock of the addictive substance. That is, if the person is not yet addicted and $S<\tilde{S}^{*}$, then $c>\delta S$ and his stock will get nearer $\tilde{S}^{*}$ as long as he is not yet addicted. (This stock is not the person's steady state since along the path he becomes addicted with some probability and the consumption pattern thereafter is completely different.)

From the earlier analysis, the following lemma is apparent:

$\underline{\text { Lemma } 3} \quad \tilde{S}^{*} \leq S^{*}$. 
Finally, we shall determine the optimal consumption by comparing the above two value functions. There are three cases.

Case 1: $S \in\left[0, \tilde{S}^{*}\right)$.

In this case, $V$ is calculated using $c(t)=\delta S(t), \forall t$, while $\tilde{V}$ is determined by $c(t)=\tilde{c}(S(t)), \forall t$. Since the former consumption path is only one of the feasible paths in the latter case, $\tilde{V}(S)$ must be at least as great as $V(S)$. Therefore, following $c(t)=\tilde{c}(S(t)), \forall t \geq 0$, is optimal.

Case 2: $S \in\left[\tilde{S}^{*}, S^{*}\right]$.

In this case, $V(S)=\tilde{V}(S)$, since $c=\delta S$ is used in both calculations. Therefore, $c=\delta S$ is the optimal consumption path.

Case 3: $S>S^{*}$.

Similarly to Case $1, \tilde{V}$ is calculated using $c=\delta S$ while $V(S)$ is calculated using $c(t)=c^{*}(S(t))$, $\forall t$. Since the former is one of the feasible paths in the latter situation, which value function is maximized at $c(t)=c^{*}(S(t)), \forall t$, the latter must be at least as good as the former. Therefore, $c(t)=c^{*}(S(t)), \forall t$, is the optimal path. 
These results are summarized in the following theorem. An illustrative sketch of the optimal consumption path is given by Figure 3 .

Theorem 1 Suppose that a person is not initially addicted at $S_{0}$. Then the optimal consumption path (given that he is not yet addicted) satisfies the following properties: if $S_{0}<\tilde{S}^{*}$, $c(t)=\tilde{c}(S(t)), \forall t$, and $S$ is increasing and getting closer and closer to $\tilde{S}^{*} ;$ if $S_{0}>S^{*}, c(t)=c^{*}(S(t))$, $\forall t$, and $S$ is decreasing and converging to $S^{*}$; and if $S_{0} \in\left[\tilde{S}^{*}, S^{*}\right], c=\delta S_{0}$ and $S$ is constant.

The intuition behind Theorem 1 is as follows. If a person has an initial stock higher than $S^{*}$, he should reduce his stock along the optimal path. This is because his stock is higher than both $\tilde{S}^{*}$ and $S^{*}$, and he should reduce his stock with or without the risk of addiction. If his initial stock is lower than $\tilde{S}^{*}$, he should increase his stock. This is because the stock is lower than both $\tilde{S}^{*}$ and $S^{*}$ and he should increase his stock with or without the risk of addiction. The intermediate case occurs if his initial stock, $S_{0}$, is between $\tilde{S}^{*}$ and $S^{*}$. The target stock in the presence of risk of addiction, $\tilde{S}^{*}$, is lower than $S_{0}$, which indicates that he should not increase his stock with the risk of addiction. On the other hand, the steady-state stock in the absence of risk of addiction $S^{*}$ is higher than $S_{0}$, which indicates that he should not reduce his stock even if there is no risk of 
addiction. Therefore, his optimal consumption is the exact amount as the depreciation, $\delta S_{0}$; and $S$ remains constant at all times.

The following implications can be deduced from the above analysis. A person who starts with zero or little stock of the addictive substance would experiment with the addictive good and increase his stock to $\tilde{S}^{*}$. As long as he has not yet reached his threshold value $\bar{S}$, he is not addicted to the good and could quit the consumption easily. Of course, if he unfortunately reaches his $\bar{S}$, he is hooked and suffers a substantial drop in his utility. Note that $\tilde{S}^{*}$ is less than $S^{*}$, which is the steady state stock the person would accumulate if there is no risk of addiction. As suggested by a referee, the difference between the two represents a solution to an optimization problem under uncertainty: "I like to rock-climb and hike along canyons and cliffs because of the utility I derive, while realizing that some danger exists; the potential for danger increases if I get too close to the edge ... I do not know with certainty exactly where the 'too close' threshold is so I leave a healthy margin for error." This healthy margin is given by the difference between $\tilde{S}^{*}$ and $S^{*}$.

For a non-addicted person who starts with a medium stock, he would consume the good at a constant rate, keeping his stock of the addictive substance unchanged. For this type of consumer, consuming more leads to an unwanted addiction with some probability, while consuming less is not utility maximizing. (In the above rock-climbing example, this person is currently in no danger and enjoys the view very much. But moving closer to the edge becomes too dangerous. Therefore, he stays where he is.) This type of consumer is not addicted either, and his consumption of the addicted good could be easily stopped. A person who is not addicted despite a large initial stock would reduce his stock to $S^{*}$. This is not a process of quitting as the person is not addicted to the good. (This climber does not enjoy the view, even though he is not in any danger.)

To conclude, a person who is not initially addicted could become addicted as he experiments with the good. If $\bar{S}<\tilde{S}^{*}$, this person will become addicted some time along the consumption path. When this occurs, the analysis of the next section applies.

\section{The Quitting Behavior}

From the analysis in Section I, we know that if $S_{0}<\tilde{S}^{*}$, a non-addicted person will consume more than the depreciation, thus $S$ increases over time. The probability of becoming addicted is given by c.d.f. $G(\cdot)$. Suppose that a person is addicted for the very first time at $t_{a}$ with a stock $\mathcal{S}_{0}=\bar{S}$ of the addictive substance. He knows perfectly his threshold level of addiction. (If the person becomes addicted at the very beginning, he does not know his $\bar{S}$. The analysis for this case is parallel.) His threshold level of quitting, $\underline{S}$, is lower than $\mathcal{S}_{0}$. He will then have to decide whether or not to initiate an attempt to quit the addiction by consuming less than the internal depreciation of the addictive substance, thus reducing his stock. 
The person's optimization problem is to find the optimal consumption path until the addiction is terminated. ${ }^{7}$ Hence,

$$
\begin{aligned}
W(\bar{S}) & \equiv \max E_{a} \int_{t_{a}}^{\infty} u(c(t), S(t), I(t)) e^{-\rho\left(t-t_{a}\right)} d t \\
& =\max E_{a}\left\{\int_{t_{a}}^{t_{q}} \mathcal{U}(c(t), S(t)) e^{-\rho\left(t-t_{a}\right)} d t+\int_{t_{q}}^{\infty} u(c(t), S(t), I(t)) e^{-\rho\left(t-t_{a}\right)} d t\right\} \\
& =\max E_{a}\left\{\int_{t_{a}}^{t_{q}} \mathcal{U}(c(t), S(t)) e^{-\rho\left(t-t_{a}\right)} d t+e^{-\rho\left(t_{q}-t_{a}\right)} \mathcal{W}(\underline{S})\right\}
\end{aligned}
$$

where the expectation is taken at $t_{a}$ conditional upon $S\left(t_{a}\right)=\bar{S}$ and $I\left(t_{a}\right)=1$.

According to Lemma 1, there are three possibilities between the person's first addiction and his first successful quitting, depending on the switching time T:

i) $S$ is always decreasing until the addiction is terminated, so $T=\infty$ in this case;

ii) $S$ is always increasing, so $T=t_{a}$ in this case; or

iii) there exists a finite $T \in\left(t_{a}, \infty\right)$, such that $S$ is decreasing for $t_{a} \leq t \leq T$ until the addiction is terminated, and increasing for $t>T$ when quitting is not yet successful by $T$.

Define $\hat{\mathcal{S}} \equiv S(T)$ as the person's switching stock. (Note that $\hat{\mathcal{S}}=0$ in case i), and $\hat{\mathcal{S}}=\infty$ in case ii).) With positive probability, the person reaches his threshold level $\underline{S}$ and quits successfully by the switching time. Before that, he would consume less than the depreciation until $T$, at which time he abandons his quitting effort and consumes more than the depreciation. Note that if we start with any stock (other than $\bar{S}$ ) which is on the quitting path $S$, the switching stock is still $\hat{\mathcal{S}}$. This is because the information the person has at $S$ is exactly the same: his quitting threshold $\underline{S}$ is lower than $S$, no matter if he arrived at that stock by trying to quit or he just became addicted at that stock.

To summarize, given that the person is addicted at $\mathcal{S}_{0}$, the person's stock $S$ is decreasing until it reaches $\hat{\mathcal{S}}$ (which could depend on the initial $\mathcal{S}_{0}$ ), at which point his $S$ starts to increase (given that he is still addicted at that time).

This property of the optimal consumption path simplifies the analysis and allows us to adopt the following approach. For each $\mathcal{S}_{0}$, we first look for the optimal path among all feasible paths corresponding to a particular $\hat{\mathcal{S}}$. Then we choose the best one among these optimal paths for each $\mathcal{S}_{0}$. From Lemma 1, this must be the overall optimal path for this $\mathcal{S}_{0}$. We shall do this in three subsections. In Subsection A, we analyze the case where $\hat{\mathcal{S}}=\infty$ (for any given $\mathcal{S}_{0}$ ). In this case,

\footnotetext{
${ }^{7}$ Once he terminates his addiction, he knows $\bar{S}$ and $\underline{S}$ perfectly. The optimal consumption path is relatively easy to solve in this case and it is again important in the quitting analysis. Nevertheless, it is not the focus of this paper, and is therefore omitted. What we need are inequalities in the values functions which are guaranteed by the assumption of harmful addiction.
} 
the person abandons his quitting effort right at the beginning. In Subsection B, we study the case

where $\hat{\mathcal{S}}=0$ (for any given $\mathcal{S}_{0}$ ). In this case, the person is always trying to quit. Finally, in Subsection $\mathrm{C}$, we look for the optimal path for each $\mathcal{S}_{0}$ by varying $\hat{\mathcal{S}}$. We find that each of these optimal paths combines parts of the constrained optimal paths in Subsections A and B. The optimal path corresponding to the optimal $\hat{\mathcal{S}}$ must be the optimal path for that $\mathcal{S}_{0}$, following directly from Lemma 1. In what follows, we shall use script letters to distinguish functions and values in this analysis from those in the previous analysis.

\section{(A) The Optimal Path Corresponding to $\hat{\mathcal{S}}=\infty$}

We first consider the case where $\hat{\mathcal{S}}=\infty$ for all $\mathcal{S}_{0}$. In this case, $S$ is increasing all the time. The person is not allowed to quit his addiction and must consume $c \geq \delta S$ at any time. Therefore, $\mathcal{U}$ becomes the only relevant utility function. We should characterize the optimal path under this constraint. Let

$$
\begin{aligned}
& \mathcal{V}\left(\mathcal{S}_{0}\right)=\max \int_{t_{0}}^{\infty} \mathcal{U}(c, S) e^{-\rho\left(t-t_{0}\right)} d t . \\
& \text { s.t. } \quad \dot{S}=c-\delta S, \quad c \geq \delta S, \quad S\left(t_{0}\right)=\mathcal{S}_{0}
\end{aligned}
$$

be the present value of the person's maximized utility with a stock of $\mathcal{S}_{0}$ at a particular $t_{0} \geq t_{a}$. We first ignore the inequality constraint of the above problem and let $\mathcal{H}=\mathcal{U}(c, S)+\mu(c-\delta S)$ be the current value Hamiltonian for the unconstrained maximization problem. We have first-order conditions $\mathcal{H}_{c}=0, \mathcal{H}_{S}=\rho \mu-\dot{\mu}$. The trajectories for the possible optimal consumption path in the $c-S$ phase diagram are characterized by the state equation $\dot{S}=c-\delta S$ and

$$
\dot{c}=\frac{1}{\mathcal{U}_{c c}}\left[(\delta+\rho) \mathcal{U}_{c}+\mathcal{U}_{S}-\mathcal{U}_{c S}(c-\delta S)\right]
$$

where (11) is obtained by solving the above mentioned first-order conditions. The steady-state stock $\mathcal{S}^{*}$ is given by the solution to $\dot{c}=0$ and $\dot{S}=0$; that is,

$$
(\delta+\rho) \mathcal{U}_{c}\left(\delta \mathcal{S}^{*}, \mathcal{S}^{*}\right)+\mathcal{U}_{S}\left(\delta \mathcal{S}^{*}, \mathcal{S}^{*}\right)=0
$$

Similar to the last section, we need the following two assumptions to guarantee that the solution to the above equation is unique with $\mathcal{S}^{*} \geq 0$, and that the system's steady state is a saddle point, therefore, that the optimal path is characterized by the saddle path. 
Assumption $3 \quad(\delta+\rho) \mathcal{U}_{c}(0,0)+\mathcal{U}_{S}(0,0) \geq 0$.

Assumption $4 \quad \delta \mathcal{U}_{c c}(c, S)+\mathcal{U}_{c S}(c, S)<0, \forall c \geq 0, \forall S \geq 0$

Again, Assumption 3 ensures that the person would prefer to consume some amount of the addictive good given that there is no chance of quitting. The person may not consume anything at all if there is some chance that he is able to quit and his utility could be increased to $U(\cdot, \cdot)$. Assumption 4 implies that $\mathcal{U}_{c}(\delta S, S)$ is a decreasing function of $S$. Together with the concavity of $\mathcal{U}$, it also implies that $(\delta+\rho) \mathcal{U}_{c}(\delta S, S)+\mathcal{U}_{S}(\delta S, S)$ is a decreasing function of $S$. (See (2) for a parallel derivation.) Together with Assumption 3, they guarantee that the solution to (12), the candidate for a steady state, is unique and non-negative. Similar to the previous section, we can argue that for the unconstrained problem, the saddle path defined as $c(t)=\mathcal{C}^{*}(S(t)), \forall t$, exists and is indeed the optimal solution. Similarly to Lemma 2, we can argue that $\mathcal{C}^{*}(\cdot)$ is continuous and differentiable. Furthermore, along this path, $c>\delta S$ (and thus $\dot{S}>0$ ) when $S<\mathcal{S}^{*}$, and $c<\delta S$ (and thus $\dot{S}<0$ ) when $S>\mathcal{S}^{*}$.

We argue that the part of $c(t)=\mathcal{C}^{*}(S(t))$ that satisfies $c(t)>\delta S(t)$ (i.e., when $S(t)<\mathcal{S}^{*}$ ), $\forall t$, is still optimal when the constraint on $c$ is imposed. This is clear because the constraint is automatically satisfied along the unconstrained optimal path.

Recall the value function defined by (10). From Stokey and Lucas (Theorem 4.8, p. 81, and Theorem 4.10, p. 84, 1989), $\mathcal{V}$ is strictly concave and differentiable.

When $S>\mathcal{S}^{*}$, the constraint is no longer satisfied along the unconstrained optimal path. Similar to the model in the previous section, we can show that the constrained optimal consumption is given by $c=\delta S$. Along this path, the person's stock remains unchanged.

We summarize the above results in the following lemma:

Lemma 4 Suppose that $c \geq \delta S$ is imposed. Then the optimal consumption path is given by the saddle path $c(t)=\mathcal{C}^{*}(S(t))$ (where $\mathcal{C}^{*}(\cdot)$ is continuous), $\forall t$, if $\mathcal{S}_{0}<\mathcal{S}^{*}$, and by $c(t)=\delta \mathcal{S}_{0}$ if $\mathcal{S}_{0} \geq \mathcal{S}^{*}$

\section{(B) The Optimal Path Corresponding to $\hat{\mathcal{S}}=0$}

When $\hat{\mathcal{S}}=0$ for a given $\mathcal{S}_{0}, S$ declines along all feasible paths and the person is continuously trying to quit. In this case, $c \leq \delta S$ holds along the consumption path, as long as the person is still 
addicted. Along the way the addiction is terminated stochastically. (Note that the optimal path we shall find is optimal for every $\mathcal{S}_{0}$, since if a person is still addicted at a particular stock, say $S$, then the information is that $\underline{S}$ is still lower than $S$, regardless of how the person had reduced his stock to $S$.)

Recall that $\mathcal{V}(S)$ is the present value of an addicted person's maximized utility with a current stock of the addictive substance $S$ when $c \geq \delta S$ is imposed. When $c \leq \delta S$ is imposed, let $\tilde{\mathcal{V}}(S)$ be the corresponding present value of the person's maximized utility with a current stock of the addictive substance $S$ (given a known $\bar{S}$ ). ${ }^{8}$

Recall that $\mathcal{W}(S)$ is the present value of the person's maximized utility if he quits the addiction exactly at $S$. In this paper, we shall not analyze and calculate $\mathcal{W}(S)$, except assuming that it is differentiable and strictly concave. What we need to describe the optimal path (qualitatively) is the following condition, which is guaranteed by our assumption of harmful addiction:

$$
\mathcal{W}(S)>\tilde{\mathcal{V}}(S), \forall S \geq 0
$$

that is, quitting successfully is always strictly better than being presently addicted and still trying to quit. For a healthy addiction, a person will never quit and thus the analysis here is not applicable.

Suppose that, along the quitting path, the person is still addicted at $t=t_{0}>t_{a}$. Using the above newly defined functions and the principle of optimality, the person's optimization problem under the constraint of $c \leq \delta S$ can be written as:

$$
\begin{aligned}
\tilde{\mathcal{V}}(S) & =\max _{c \leq \delta S} E_{0} \int_{t_{0}}^{\infty} u(c(t), S(t), I(t)) e^{-\rho\left(t-t_{0}\right)} d t \\
& =\max _{c \leq \delta S} E_{0}\left\{\int_{t_{0}}^{t_{q}} \mathcal{U}(c(t), S(t)) e^{-\rho\left(t-t_{0}\right)} d t+e^{-\rho\left(t_{q}-t_{0}\right)} \mathcal{W}(\underline{S})\right\}
\end{aligned}
$$

where the expectation is taken conditional upon $S\left(t_{0}\right)=S, t_{0} \geq t_{a}$, and $I\left(t_{0}\right)=1$. From Stokey and Lucas (Theorem 9.8, p. 265, and Theorem 9.10, p. 266, 1989), $\tilde{\mathcal{V}}$ is strictly concave and continuously differentiable.

Suppose that the person has not yet reached $\underline{S}$ by $t$. The probability that he will reach $\underline{S}$ by $t+d t$ is $\frac{F(S(t))-F(S(t+d t))}{F(S(t))}$. Similar to (6), the solution to this person's dynamic optimization problem is given by the following Hamilton-Jacobi-Bellman equation:

$$
\begin{array}{r}
\tilde{\mathcal{V}}(S(t)) e^{-\rho t}=\max _{c(t) \leq \delta S(t)}\left\{\mathcal{U}(c(t), S(t)) e^{-\rho t} d t+\left(1+\frac{f(S(t))}{F(S(t))} d S(t)\right) \tilde{\mathcal{V}}(S(t+d t)) e^{-\rho(t+d t)}\right. \\
\left.-\frac{f(S(t))}{F(S(t))} d S(t) \mathcal{W}(S(t+d t)) e^{-\rho(t+d t)}\right\},
\end{array}
$$

\footnotetext{
${ }^{8}$ We omit the argument $\bar{S}$ in this and other value functions since it does not cause any confusion. Note that $\tilde{\mathcal{V}}(\bar{S})=W(\bar{S})$.
} 
where $-\frac{f(S(t))}{F(S(t))} d S(t)$ is the conditional probability that the person quits the addiction between $t$ and $t+d t$ (and gets $\mathcal{W}(S(t+d t))) \cdot{ }^{9}$ Otherwise, the person gets $\tilde{\mathcal{V}}(S(t+d t))$ again (with probability $\left.1+\frac{f(S(t))}{F(S(t))} d S(t)\right)$. Therefore, the right-hand side of the above equation represents the utility of consuming $c(t)$ for a very short period of time (from $t$ to $t+d t$ ).

Rearranging (13), ignoring higher-order terms, and making use of $\frac{d S}{d t}=c-\delta S, e^{-\rho d t}=1-\rho d t$, $\tilde{\mathcal{V}}(S(t+d t))=\tilde{\mathcal{V}}(S(t))+\tilde{\mathcal{V}}^{\prime}(S(t)) S^{\prime}(t) d t$ and $\mathcal{W}(S(t+d t))=\mathcal{W}(S(t))+\mathcal{W}^{\prime}(S(t)) S^{\prime}(t) d t$, we have

$$
\rho \tilde{\mathcal{V}}(S)=\max _{c \leq \delta S}\left\{\mathcal{U}(c, S)+\tilde{\mathcal{V}}^{\prime}(S)(c-\delta S)-\frac{f(S)}{F(S)}[\mathcal{W}(S)-\tilde{\mathcal{V}}(S)](c-\delta S)\right\} .
$$

The first-order condition for the optimal $c(t)$ obtained from the maximization in (14) is given by:

$$
\mathcal{U}_{c}(c, S)+\tilde{\mathcal{V}}^{\prime}(S)-\frac{f(S)}{F(S)}[\mathcal{W}(S)-\tilde{\mathcal{V}}(S)]=0
$$

The sufficient second-order condition is always satisfied, because $\mathcal{U}(\cdot, \cdot)$ is strictly concave. Note that the consumption function derived from the above equation, denoted by $c(t)=\tilde{\mathcal{C}}(S(t)), \forall t$, is valid if and only if the person is currently addicted. Note that each term in (15) is continuous, and that $\mathcal{U}_{c}$ is differentiable. It is easy to see that $\tilde{\mathcal{C}}(\cdot)$ is continuous.

Let $\tilde{\mathcal{S}}^{*}$ be the target stock along this quitting path; that is, $S$ will get nearer to $\tilde{\mathcal{S}}^{*}$ along $c(t)=\tilde{\mathcal{C}}(S(t))$ if the person attempts to quit but not yet terminate the addiction. To find this target stock, we differentiate (14) with respect to $S$ along the optimal path $c$, and evaluate it at $c=\delta S$. We have

$$
\rho \tilde{\mathcal{V}}^{\prime}(S)=\mathcal{U}_{S}(\delta S, S)-\delta \tilde{\mathcal{V}}^{\prime}(S)+\delta \frac{f(S)}{F(S)}[\mathcal{W}(S)-\tilde{\mathcal{V}}(S)]
$$

Combining (15) and (16) and evaluating at the target stock $\tilde{\mathcal{S}}^{*}$ yields

$$
\mathcal{U}_{c}\left(\delta \tilde{\mathcal{S}}^{*}, \tilde{\mathcal{S}}^{*}\right)+\frac{1}{\delta+\rho} \mathcal{U}_{S}\left(\delta \tilde{\mathcal{S}}^{*}, \tilde{\mathcal{S}}^{*}\right)=\frac{\rho}{\delta+\rho} \frac{f\left(\tilde{\mathcal{S}}^{*}\right)}{F\left(\tilde{\mathcal{S}}^{*}\right)}\left[\mathcal{W}\left(\tilde{\mathcal{S}}^{*}\right)-\tilde{\mathcal{V}}\left(\tilde{\mathcal{S}}^{*}\right)\right]
$$

The right-hand side of (17) is positive because of the assumption of harmful addiction. Similarly to (2), we can show that the left-hand side of (17) is strictly decreasing in $S$ because of Assumption 4 and the concavity of $\mathcal{U}(\cdot, \cdot)$. Comparing (17) with (12), and noting that the right-hand side of (17) is strictly positive, we can conclude immediately that $\tilde{\mathcal{S}}^{*}<\mathcal{S}^{*}$, which is stated in the following lemma:

Lemma $5 \quad \tilde{\mathcal{S}}^{*}<\mathcal{S}^{*}$.

\footnotetext{
${ }^{9}$ Note that this probability is positive, since $d S$ is negative here.
} 
As the level of utility after quitting the addiction is higher, the person has more incentive to reduce his stock than in the case where he always gets the lower level utility, hoping to quit the addiction successfully. Therefore, it is not surprising that the target stock in the case of quitting must be less than $\mathcal{S}^{*}$, which is the steady state when there is no hope of quitting.

The path determined by (15) is indeed an optimal path under the constraint $c \leq \delta S$ if the path indeed satisfies this constraint, which is true when $S \geq \tilde{\mathcal{S}}^{*}$. When $S<\tilde{\mathcal{S}}^{*},(15)$ implies that $c>\delta S$. We argue that in this case, the corner solution $c=\delta S$ is the constrained optimal path. This is because $\mathcal{U}$ is strictly concave in $c$. Therefore, $\mathcal{U}_{c}$ is decreasing in $c$. If the left-hand side of (15) is zero at $c>\delta S$, then it is positive for all $c \leq \delta S$, which implies that $c=\delta S$ is indeed optimal under the constraint $c \leq \delta S$.

We summarize the above results in the following lemma:

Lemma 6 Suppose that $\hat{\mathcal{S}}=0$. Then $c \leq \delta S$ must be satisfied along all feasible paths. The optimal path is given by $c(t)=\tilde{\mathcal{C}}\left(S(t)\right.$ ) (where $\tilde{\mathcal{C}}$ is continuous), $\forall t$, if $\mathcal{S}_{0} \geq \tilde{\mathcal{S}}^{*}$, and by $c(t)=\delta \mathcal{S}_{0}$ if $\mathcal{S}_{0}<\tilde{\mathcal{S}}^{*}$.

\section{(C) The Unconstrained Optimal Path For a Particular $\mathcal{S}_{0}$}

With the analysis in Subsections A and B, we can now characterize the unconstrained optimal consumption path for any particular $\mathcal{S}_{0}$ by varying $\hat{\mathcal{S}}$. The optimal path we find must be the optimal path for the particular $\mathcal{S}_{0}$, since the optimal path must be represented by some $\hat{\mathcal{S}}$. In the process, we are able to find an $\hat{\mathcal{S}}$ that is the common switching stock for all $\mathcal{S}_{0}>\hat{\mathcal{S}}$.

Given $\mathcal{S}_{0}$ and $\hat{\mathcal{S}}$, a feasible path must satisfy the following condition: if $\mathcal{S}_{0}<\hat{\mathcal{S}}, c(t) \geq \delta S(t)$, $\forall t$; if $\mathcal{S}_{0} \geq \hat{\mathcal{S}}$, then $c(t) \leq \delta S(t)$ (if the person is still addicted at the time) until $S(t)$ reaches $\hat{\mathcal{S}}$ for the first time, and then $c(t) \geq \delta S(t)$ for the rest of the path. We divide $\mathcal{S}_{0}$ into three cases:

Case $1: \mathcal{S}_{0}<\tilde{\mathcal{S}}^{*}$.

If we set $\hat{\mathcal{S}} \geq \mathcal{S}_{0}$, it is equivalent to setting $\hat{\mathcal{S}}=\infty$, since $S$ will decrease to $\hat{\mathcal{S}}$ monotonically. Therefore, $S$ must be monotonically increasing all the time (c.f. Lemma 1) and $c(t)=\mathcal{C}^{*}(S(t)), \forall t$, is optimal.

If we set $\hat{\mathcal{S}}<\mathcal{S}_{0}$, then $S$ is decreased to $\hat{\mathcal{S}}$ at first and, if the person is still addicted by the time $S$ reaches $\hat{\mathcal{S}}, S$ will be increased. Note that $\tilde{\mathcal{S}}^{*}$ is the target stock for quitting. Below that level of stock, the person prefers $c=\delta S$ (and keeping a constant stock) to quitting (which is evident 
from the fact that the left-hand side of (15) is positive for $c=\delta S$ given $S<\tilde{\mathcal{S}}^{*}$ ). Furthermore, $c(t)=\mathcal{C}^{*}(S(t)), \forall t$, dominates $c(t)=\delta S(t), \forall t$ (obtained in the case where the person is not allowed to quit). Hence, $c(t)=\mathcal{C}^{*}(S(t)), \forall t$, (corresponding to $\hat{\mathcal{S}}=\infty$ ) must be a better path than any path corresponding $\hat{\mathcal{S}}<\mathcal{S}_{0}$.

To summarize, $c(t)=\mathcal{C}^{*}(S(t)), \forall t$, is optimal path when $\mathcal{S}_{0}<\tilde{\mathcal{S}}^{*}$.

Case 2: $\mathcal{S}_{0}>\mathcal{S}^{*}$.

There is no doubt that following the quitting path $c(t)=\tilde{\mathcal{C}}(S(t)), \forall t$, is optimal. This is because $c=\delta S$ is optimal if we impose $c \geq \delta S$. But it is dominated by the quitting path (obtained in the case where we impose $c \leq \delta S$ ). The selection of the optimal $\hat{\mathcal{S}}$ is discussed in the next case, as we have a problem identical to the one with $\mathcal{S}_{0}=\mathcal{S}^{*}$ when $S$ reaches $\mathcal{S}^{*}$.

$\underline{\text { Case } 3:} \mathcal{S}_{0} \in\left[\tilde{\mathcal{S}}^{*}, \mathcal{S}^{*}\right]$.

We have established in Case 1 that $\mathcal{V}(S)>\tilde{\mathcal{V}}(S)$ (the non-quitting path is better) when $S<\tilde{\mathcal{S}}^{*}$, and in Case 2 that the opposite is true when $S>\mathcal{S}^{*}$. By the continuity and concavity of $\mathcal{V}(\cdot)$ and $\tilde{\mathcal{V}}(\cdot)$, we know that there exists a unique $S$ such that $\mathcal{V}(S)=\tilde{\mathcal{V}}(S)$. Denote this crossing point of the two curves (c.f. Figure 4 ) as the optimal $\hat{\mathcal{S}}$, since it is the optimal switching stock for all $\mathcal{S}_{0}>\hat{\mathcal{S}}$.

For $\mathcal{S}_{0}<\hat{\mathcal{S}}, \mathcal{V}(S(t))>\tilde{\mathcal{V}}(S(t)), \forall t$. Therefore, following the non-quitting path is better. $S$ increases and eventually converges to $\mathcal{S}^{*}$. Note that in this case at $S>\hat{\mathcal{S}}$, it is not optimal for the person to switch to the quitting path, because the information he has on $\underline{S}$ (i.e., $\underline{S}<\mathcal{S}_{0}$ ) is different than the assumption in calculating $\tilde{\mathcal{V}}(S)$ (i.e., $\underline{S}<S$ ).

For $\mathcal{S}_{0}>\hat{\mathcal{S}}, \mathcal{V}(S(t))<\tilde{\mathcal{V}}(S(t)), \forall t$. Therefore, following the quitting path is better. As $S$ decreases to $\hat{\mathcal{S}}$, the person is indifferent between following the quitting path and following the non-quitting path. But for any $S<\hat{\mathcal{S}}$, the non-quitting path is certainly better. ${ }^{10}$ Therefore, the optimal strategy is to abandon his quitting effort and switch from the quitting path to the nonquitting path at exactly $\hat{\mathcal{S}}$ (given that he is still addicted at that time) and $S$ gradually converges to $\mathcal{S}^{*}$.

We summarize the above results in the following theorem:

\footnotetext{
${ }^{10}$ As a technical note, even though $\tilde{\mathcal{V}}$ is calculated based on the assumption that the person follows the quitting path from the beginning to the end, it makes no difference to the value function for $S>\hat{\mathcal{S}}$ if he switches to the non-quitting path at $\hat{\mathcal{S}}$, as $\tilde{\mathcal{V}}(\hat{\mathcal{S}})=\mathcal{V}(\hat{\mathcal{S}})$. However, if the person continues to follow the quitting path when $S<\hat{\mathcal{S}}$ and discovers that he is still addicted, then following the non-quitting path is optimal. There is no contradiction here because with some probability the person may terminate the addiction successfully along the path.
} 
Theorem $2 \quad$ a) $\mathcal{V}(S)>\tilde{\mathcal{V}}(S)$ for $S<\hat{\mathcal{S}}$ and $\mathcal{V}(S)<\tilde{\mathcal{V}}(S)$ for $S>\hat{\mathcal{S}}$.

b) For $\mathcal{S}_{0} \leq \hat{\mathcal{S}}$, the optimal consumption path is given by $c(t)=\mathcal{C}^{*}(S(t)), \forall t$, and $S$ converges to $\mathcal{S}^{*}$; for $\mathcal{S}_{0}>\hat{\mathcal{S}}$, the optimal consumption path is given by $c(t)=\tilde{\mathcal{C}}(S(t)), \forall t$, until $S$ reaches $\hat{\mathcal{S}}$ (given that he is still addicted) and then switch to $c(t)=\mathcal{C}^{*}(S(t)), \forall t$.

This theorem shows that no one would follow the quitting path from the beginning to the end. In the quitting process, a person reduces his consumption of the addictive good because of the benefit of successful quitting. The probability of attaining this benefit diminishes as the stock reaches its target. At some point, it becomes not worthwhile to sacrifice the distortion in current utility in continuing the process and the quitting effort is abolished.

The saddle paths in Figure 5 illustrate the optimal consumption paths. When $S<\hat{\mathcal{S}}, \tilde{\mathcal{V}}(S)<$ $\mathcal{V}(S)$, and the upper saddle path is followed. When $S>\hat{\mathcal{S}}, \tilde{\mathcal{V}}(S)>\mathcal{V}(S)$, and the lower saddle path is followed (as long as he is still addicted) up to $\hat{\mathcal{S}}$; after that, the person abandons his quitting effort and switches to the upper saddle path. A person may have the same stock when he follows the upper saddle path as when he follows the lower saddle path, but the information on $\underline{S}$ is different. When he follows the upper saddle path after he abandons his quitting effort, he knows that $\underline{S}<\hat{\mathcal{S}}$, while when he follows the lower saddle path he knows only that $\underline{S}<S$. 


\section{(D) Comparing the Steady States in the Quitting and the Consumption Models ${ }^{11}$}

In the previous sections, the consumption and the quitting of the addictive good were, for the most part, examined separately. These analyses can be done mainly due to the assumption that addiction is a state of harm or sickness; once a person gets into addiction, his utility level drops immediately. We never compare how much of the addictive good a person would optimally consume in the states of addiction and non-addiction. While the results obtained so far are intuitive, the interpretation of addiction is not complete unless an addicted person consumes more than a non-addicted person in the steady state. This property is not obtained from our analysis, and in fact it should not always be true given our assumptions so far, since these assumptions do not actually compare the marginal utility of consumption in the two different states. To capture the fact that an addicted person always craves for more of the addictive good, we make the following two additional assumptions.

\footnotetext{
${ }^{11} \mathrm{I}$ am indebted to a referee for his/her insights on this issue, which resulted in this subsection.
} 
Assumption $5 \quad(\delta+\rho) \mathcal{U}_{c}(0,0)+\mathcal{U}_{S}(0,0)>(\delta+\rho) U_{c}(0,0)+U_{S}(0,0)$.

Assumption $6 \quad \forall S \geq 0$,

$$
\begin{aligned}
& \mathcal{U}_{c S}(\delta S, S)+\frac{1}{2 \delta+\rho}\left[(\delta+\rho) \delta \mathcal{U}_{c c}(\delta S, S)+\mathcal{U}_{S S}(\delta S, S)\right] \\
\geq & U_{c S}(\delta S, S)+\frac{1}{2 \delta+\rho}\left[(\delta+\rho) \delta U_{c c}(\delta S, S)+U_{S S}(\delta S, S)\right]
\end{aligned}
$$

Assumption 5 states loosely that an addicted person enjoys more of the addicted good than a non-addicted person at $S=0$. Assumption 6 states that the marginal utility of consumption with a higher stock of the addictive substance is higher for an addicted person (after some normalizations of the utility function). These two assumptions together guarantee that the steady state of the addictive substance for a non-addicted person is lower than an addicted person. We have the following lemma:

Lemma $7 \quad S^{*}<\mathcal{S}^{*}$.

Proof Assumption 5 implies that $(\delta+\rho) \mathcal{U}_{c}(\delta S, S)+\mathcal{U}_{S}(\delta S, S)$ is higher than $(\delta+\rho) U_{c}(\delta S, S)+$ $U_{S}(\delta S, S)$ at $S=0$. (They are both positive, from Assumptions 1 and 3.) Meanwhile, Assumption 6 implies that the former decreases more slowly than the latter. Therefore, the latter reaches zero faster than the former. Recall (4) and (12). We conclude that $S^{*}<\mathcal{S}^{*}$.

In a steady state the person consumes the depreciation amount; that is, $c=\delta S^{*}$ or $c=\delta \mathcal{S}^{*}$. The above lemma implies that in the long run, an addicted person consumes more than a nonaddicted person. This does not warrant, however, that an addicted person always consumes more than a non-addicted person. This is because an addicted person may try to quit his addiction at first. During this quitting period, an addicted person obviously consumes less. Of course, when this person abandons his quitting effort, his consumption eventually exceeds a non-addicted person.

\section{Further Remarks}

In our model, a person switches his preference (i.e. utility function) when he becomes addicted or terminates the addiction. These utility changes are expected by the person from the very beginning. Other researchers have investigated the situation where these changes are unexpected, e.g. where an individual has conflicting preferences which are in temporary control from time to 
time. These include Winston (1980), Elster (1984), Schelling (1984), and Glazer and Weiss (1991). The implications of these models are somewhat different than ours. For example, it is often observed that people may try to quit smoking by making it more difficult to obtain a cigarette. This kind of voluntary restriction can be utility improving in their models, but is never optimal in any rational expectation model (including ours). ${ }^{12}$

Sometimes people addicted to alcohol attend Alcoholics Anonymous or other health clinics to obtain help in quitting. Unfortunately, these quitting programs provide a mixed signal to the public. Easy quitting certainly helps the addicted consumers, but it also provides incentives for other people to start consuming the good and for existing consumers to increase their consumption. As a result, the effects of these programs on the total number of consumers and on the amount of addictive goods consumed are ambiguous.

In the previous section, a person attempted to quit only once. It is often observed, however, that many people attempt to quit several times in their lives, with some eventually succeeding and others failing. This can be explained by our model, provided that new information arrives from time to time. Some possible reasons why many people try to quit again could be, for example, cancer killing a close relative, or publication of new medical research results. New information changes the person's utility function and the perceived risk or the consequences of consuming the addictive good. If we treat $\mathcal{U}(\cdot, \cdot)$ as the expected utility, then a piece of bad news reduces $\mathcal{U}(\cdot, \cdot)$. Therefore, the difference between $\mathcal{V}(\cdot)$ and $\mathcal{W}(\cdot)$ becomes larger. As the benefit of quitting increases, the person who stopped trying to quit may try again. As a result, a person may try to quit several times in his life. The timing, according to our model, must coincide with the arrival of bad news.

Of course, if the person is in the process of quitting and some good news arrives and the addictive good is not as harmful as he thought, the person may stop the quitting attempt and resume the consumption of the good. Or if the person faces a second shock after he quits, he may go back to the addictive good as well.

In both the addiction stage and the quitting stage, a person may have regret, as more information is obtained along the optimal consumption path. For example, a person may regret his initial consumption of the addictive good if he learns that he has a low $\bar{S}$. This can only occur in a model with imperfect information. A person who is trying to quit may also regret doing so because he finds out that his $\underline{S}$ is very low and quitting is too difficult. A person who does not succeed in quitting may regret it if he later finds out that a little more effort would have produced success. Therefore, the criticism that addicts are happy ${ }^{13}$ does not apply here. In our model, if a person

\footnotetext{
${ }^{12}$ There is a similar implication in Geanakoplos (1990) where an individual may forget what he has learned.

${ }^{13}$ Winston's (1980) criticism on Stigler and Becker's (1977) and thus also on Becker and Murphy's (1988) models is one example.
} 
becomes addicted after the initial consumption, he is unhappy about it and regrets having done so.

Of course, ex ante, the person may still be better off by consuming the addictive good.

\section{Conclusions}

In this paper we have presented a model of rational addiction and quitting. As a person experiments with the addictive good, he may find himself addicted. Trying to quit may then become part of utility maximizing behavior. As new information about a person's utility (most likely, the health consequences of addiction) arrives, a person who has stopped trying to quit may attempt to quit again. As people are generally imperfectly informed about their ability to resist an addictive good or the difficulty of quitting, regret can occur along the optimal path of consumption, as more information is obtained. We also argue that the effects of quitting programs are ambiguous.

Because a person's consumption directly affects his stock of the addictive substance he accumulates and thus the information about $\bar{S}$ and $\underline{S}$, the analysis would have been very complicated. Fortunately, we are able to prove that there is some monotonicity in the person's consumption patterns along the optimal path. Therefore, in both parts of the analysis, we can investigate the problem by studying the case of a monotone increasing $S$ and the case of a monotone decreasing $S$ separately, since those are the only candidates for the optimal path. The optimal consumption path can then be easily found by comparing the relative value functions at each $S$. Without the monotonicity property, the above approach would have been impossible to adopt.

The theory of rational addiction and rational quitting can also be used to explain the persistence of harmful habits and their termination. Habits and hobbies such as overeating, overworking, television watching, stamp collecting, or nail biting can be regarded as addictions. The origin of these types of behaviors may be exogenous and their stock accumulates and depreciates. Some people argue that smokers are not addicted to the nicotine in the cigarettes but to the habit of smoking. (See Viscusi (1990) for a related discussion.) The model in this paper applies here. Some existing research on habit formation (e.g., Pollak (1970, 1976), von Weisacker (1971) and Phlips (1974)) assumes that consumers are myopic. Our model shows how addiction and the persistence of harmful habits can be analyzed in rational utility-maximization models with imperfect information.

We used the concept of cumulative risk in the analysis. In addition to its use in defaultable bonds, that concept can be applied to the case of machine maintenance. The probability of a machine breaking down and generating a lower profit depends on the machine's current state, which can be improved by regular maintenance. It can also be applied to a government's credit rating. An unknown deficit level will cause an upgrade or a downgrade of the country's credit rating. The government can increase or reduce its deficit by adjusting its spending. Of course, future research must be undertaken to explore these applications. 


\section{REFERENCES}

Akerlof, G. A., 1991, Procrastination and obedience, American Economic Review 81, 1-19.

Barthold, T. A. and H. M. Hochman, 1988, Addiction as extreme-seeking, Economic Inquiry XXVI, 89-106.

Becker, G. S., M. Grossman and K. M. Murphy, 1991, Rational addiction and the effect of price on consumption, American Economic Review 81, 237-241.

Becker, G. S. and K. M. Murphy, 1988, A theory of rational addiction, Journal of Political Economy 96, 675-700.

Bordley, R. F., 1986, Satiation and habit persistence (or the Dieter's dilemma), Journal of Economic Theory $38,178-184$.

Chaloupka, F., 1991, Rational addictive behavior and cigarette smoking, Journal of Political Economy $99,722-742$.

Dasgupta, P.S. and G.M. Heal, 1974, The optimal depletion of exhaustible resources, Review of Economic Studies, Symposium, 3-28.

Dockner, E. and G. Feichinger, 1993, Cyclical consumption patterns and rational addiction, American Economic Review 83, 256-263.

Duffie, D. and K.J. Singleton, 1998, Modeling term structures of defaultable bonds, Working paper, Stanford University Graduate School of Business.

Elster, J., 1984, Ulysses and the sirens: Studies in rationality and irrationality (revised ed.) (Cambridge University Press, Cambridge).

Geanakoplos, J., 1990, Game theory without partitions, and applications to speculation and consensus, Working paper, Yale University Economics Department.

Glazer, J. and A. Weiss, 1991, Conflicting preferences and voluntary restrictions on choices, Work- 
ing paper, Boston University Economics Department.

Iannaccone, L. R., 1986, Addiction and satiation, Economic Letters 21, 95-99.

Leonard, D., 1989, Market behavior of rational addicts, Journal of Economic Psychology 10, 117144.

Leonard, D., and Van Long, N., 1992, Optimal Control Theory and Static Optimization in Economics (Cambridge University Press, Cambridge).

Michaels, R. J., 1988, Addiction, compulsion, and the technology of consumption, Economic Inquiry XXVI, 75-88.

Nickell, S.J., 1978, The investment decisions of firms (James Nisbet \& Co. Ltd, Oxford).

Orphanides, A. and Zervos, D., 1995, Rational addiction with learning and regret, Journal of Political Economy 103, 739-758.

Phlips, L., 1974, Applied consumption analysis (North-Holland, Amsterdam).

Pollak, R. A., 1970, Habit formation and dynamic demand functions, Journal of Political Economy $78,745-763$.

Pollak, R. A., 1976, Habit formation and long-run utility functions, Journal of Economic Theory 13, 272-297.

Rogers, L.C.G. and D. Williams, 1994, Diffussions, Markov processes, and martingales, Vol. 1: Foundations, 2nd ed. (John Wiley \& Sons, Chichester) .

Rogers, L.C.G. and D. Williams, 1987, Diffussions, Markov processes, and martingales, Vol. 2: Itô calculus (John Wiley \& Sons, Chichester).

Schelling, T. C., 1984, Choice and consequence (Harvard University Press, Boston).

Seierstad, K. and Sydsæter, A., 1987, Optimal control theory with economic applications, Advance 
textbooks in economics (Elsevier Science Publisher's B.V., Netherlands).

Spinnewyn, F., 1981, Rational habit formation, European Economic Review 15, 91-109.

Stigler, G. J. and G. S. Becker, 1977, De gustibus non est disputandum, American Economic Review 67, 76-90.

Stokey, N. L. and R. E. Lucas, Jr., 1989, Recursive methods in economic dynamics (Harvard University Press, Cambridge).

Viscusi, W. K., 1990, Do smokers underestimate risks? Journal of Political Economy 98, 1253-1269.

von Weisacker, C. C., 1971, Notes on endogeneous change of tastes, Journal of Economic Theory $3,345-372$.

Winston, G. C., 1980, Addiction and backsliding: a theory of compulsive consumption, Journal of Economic Behavior and Organization 1, 295-324.

Yaari, M., 1965, Uncertain lifetime, life insurance and the theory of the consumers, Review of Economic Studies 32, 137-150. 